\title{
MATHEMATICAL MODELING OF THERMAL ABLATION TREATMENTS IN HEART ARRHYTHMIAS
}

\author{
AITOR AMATRIAIN ${ }^{1}$, IGNACIO PARRA ${ }^{1,2}$, GONZALO RUBIO $^{1,2}$ \\ AND EUSEBIO VALERO ${ }^{1,2}$ \\ ${ }^{1}$ ETSIAE-UPM - School of Aeronautics, Universidad Politécnica de Madrid, \\ Plaza Cardenal Cisneros 3, 28040, Madrid, Spain \\ ${ }^{2}$ Center of Computational Simulation, Universidad Politécnica de Madrid, \\ Campus de Montegancedo, Boadilla del Monte, 28660, Madrid, Spain
}

Key words: Arrhythmia, Catheter Ablation, Cardiac Tissue, Temperature Distribution, Heart Model

\begin{abstract}
In this work we develop a model of catheter ablation based on electromagnetic and thermal equations. This model allows the computation of the space and time evolution of temperature in the surroundings of the ablation zone. This result is relevant, as excessive temperature values may cause stream pops or esophageal ulcers. The resulting system of equations is solved using a Chebyshev spectral collocation method. Special attention is paid to the effect of blood perfusion and electrical conductivity, as there is no consensus in the literature concerning the modeling of these terms. In order to obtain the conditions that give rise to safer ablations, a parametric study is performed, where the effect of discharge time, discharge voltage and size of the electrode in the temperature distribution is analysed.
\end{abstract}

\section{INTRODUCTION}

Myocardial contraction and relaxation are produced through electrical impulses, which are transmitted by means of pacemaker cells, as part of the cardiac conduction system. If they do not work correctly spurious oscillations can be produced, which can lead to cardiac diseases as arrhythmias.

An arrhythmia is a disorder of the heart rhythm. There exist different types of arrhythmias, depending on the affected zone and the changes in the heart rate, even though the most common one is atrial fibrillation. Its prevalence in 2010 ranged from 2.7 to 6.1 million people in the USA, while in the European Union it was estimated that it was present in 8.8 million adults older than 55 years [1]. These numbers rise every year as a consequence of the increase in life expectancy and the improvements in detection methods.

Catheter ablation is a procedure to treat atrial fibrillation, in which a catheter is introduced into the femoral vein, to then be advanced into the heart through the femoral vein and the inferior vena cava. The process is monitorised at all times by the doctors, and the affected zone is identified by means of a three dimensional map of the electrical activity of the heart. Once the catheter is placed there, an electric shock is applied in order to destroy the abnormal tissue.

Despite the fact that catheter ablation has been recommended by experts in 2012 guidelines [2], the procedure has several risks. If the temperature of the cardiac tissue is around $100{ }^{\circ} \mathrm{C}$, stream pops may cause cardiac perforation [3]. The reduced distance between the endocardium and the esophagus, 
which is around $5 \mathrm{~mm}$ [4], poses an additional risk, as ulcers can be produced if the temperature of the esophagus is greater than $40{ }^{\circ} \mathrm{C}$ [5]. Currently, luminal temperature sensors are used, even though the maximum admisible response time of the sensors should be limited to $4 \mathrm{~s}$ [6], and this condition is not met by all of the current models [7]. Furthermore, luminal sensors do not to control esophageal damage completely, as greater temperature values can be achieved in the walls of the esophagus [8].

In regards to numerical simulation of catheter ablation, first studies have been based on the development of two dimensional models, considering different thermal conductivities and catheter lengths [9]. Three dimensional axisymmetric geometries have also been considered, and the influence of myocardial properties [10] and ablator material [11] has been analysed. However, in these cases Robin boundary conditions are proposed at catheter-tissue and tissue-blood interfaces, and the values of heat transfer coefficients have not been validated. Numerical simulations in three dimensional geometries have been also carried out [12], even though the obtained maximum temperatures are greater than $100{ }^{\circ} \mathrm{C}$, so the proposed cases carry a risk in terms of cardiac perforation. A recent work has proposed a more advanced model [13], although the associated computational cost is high, as the mesh requires $\sim 10^{6}$ elements. In addition, to the authors knowledge, only one work takes into account the presence of the esophagus [14].

In view of the above, the main goal of this work is to give a first step towards a reliable and affordable model of catheter ablation, in order to study the risks of the procedure. This will be done avoiding the use of Robin boundary conditions, analysing the importance of the modeling of some terms of the model and focusing attention on the damage to the esophagus. To that end, first of all, a mathematical model is developed in Section 2, giving rise to a simplified system of equations that is solved numerically using

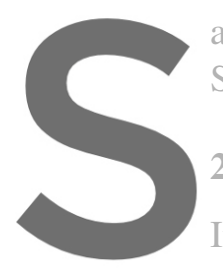

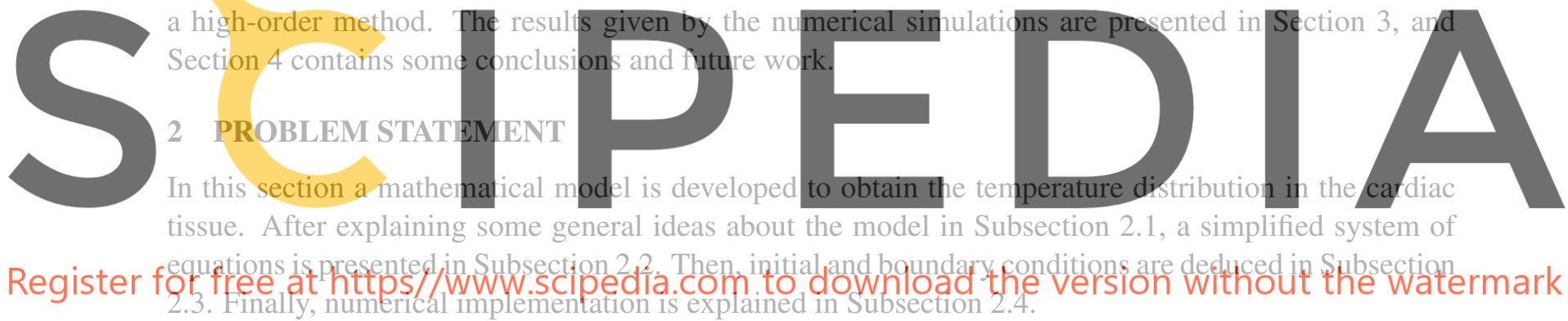

\subsection{Overview of the model}

As previously mentioned, catheter ablation consists of applying radiofrequency energy. This process is governed by Maxwell equations and Ohm's law. Moreover, resistors dissipate energy that leads to temperature increase in the surroundings of the ablator, which is modeled by energy equation. This system of equations is the starting point of the model.

The parameters of the problem vary between the cardiac tissue and the blood, so the domain of the problem is split in two parts: $\Omega=\Omega_{1} \cup \Omega_{2}$. The cardiac tissue is identified with $\Omega_{1}$, while $\Omega_{2}$ defines the zone where blood is present. The catheter is approximated as a sphere of radius $\varepsilon$, and each of the subdomains is modeled a semi-spherical shell limited by the surface of the catheter, and $r\left(\Omega_{i \infty}\right) \rightarrow \infty, i=$ 1,2 (see Figure 1). Moreover, rotational symmetry is assumed $(\partial / \partial \psi=0)$ and three spatial coordinates are considered: radial coordinate $r \in[\varepsilon, \infty)$ and polar coordinates $\theta, \varphi \in[0, \pi / 2)$.

Furthermore, it is assumed that the ablation is performed near pulmonary veins [15], so as a first approximation blood velocity is assumed to be constant in magnitude and direction, $\vec{v}=-v \vec{r}$. 


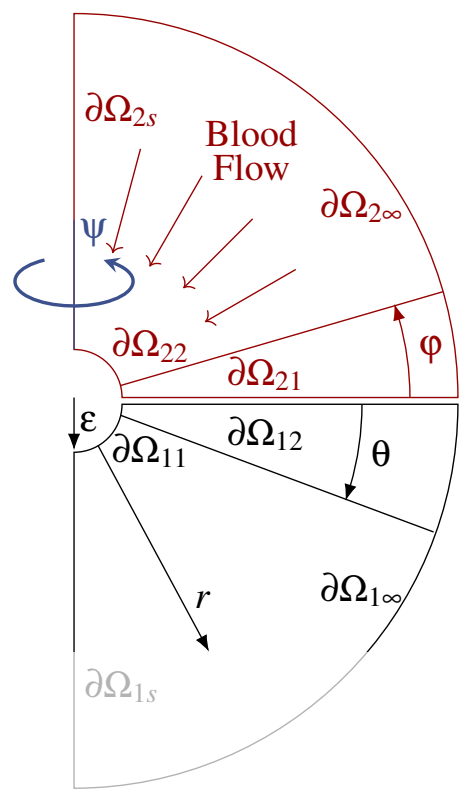

Figure 1: Domain of the problem

\subsection{Equations}

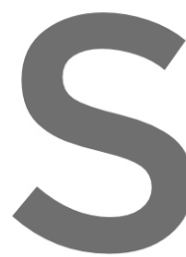

First of all, Maxwell equati
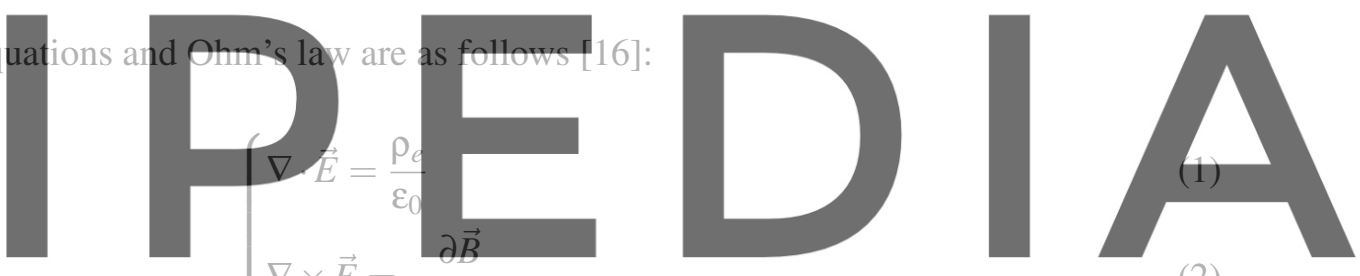

(2)

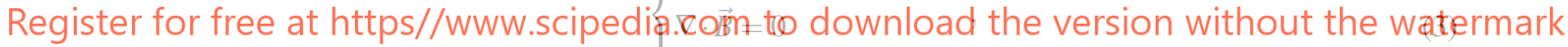

$$
\left\{\begin{array}{l}
\nabla \times \vec{B}=\mu_{0}\left(\vec{J}+\varepsilon_{0} \frac{\partial \vec{E}}{\partial t}\right) \\
\vec{J}=\sigma(\vec{E}+\vec{v} \times \vec{B})
\end{array}\right.
$$

where $\vec{E}$ is electric field, $\rho_{e}$ charge density, $\vec{B}$ magnetic field, $\vec{J}$ current density, $\vec{v}$ velocity, $t$ time, $\sigma$ electrical conductivity, $\mu_{0}=4 \pi \cdot 10^{-7} \mathrm{H} / \mathrm{m}$ vacuum permeability and $\varepsilon_{0}=1 /\left(\mu_{0} \mathrm{c}^{2}\right) \mathrm{F} / \mathrm{m}$ vacuum permittivity, where $c$ is the speed of light. The previous system of equations is completed with energy equation [17]:

$$
\rho \frac{\partial e}{\partial t}+\rho \vec{v} \cdot \nabla e=-\nabla \cdot \vec{q}-p \nabla \cdot \vec{v}+\phi_{v}+\vec{J} \cdot \vec{E}+Q,
$$

being $\rho$ density, $e$ internal energy, $\vec{q}$ heat flux, $p$ pressure, $\phi_{v}$ viscous dissipation function and $Q$ heat source. In order to obtain a simplified system of equations, the following assumptions are made:

- A low-frequency approximation is applied to Maxwell equations, so $\partial \vec{B} / \partial t=\partial \vec{E} / \partial t=0$. This 
is because $\varepsilon_{0} \omega / \sigma_{c} \ll 1$ and $\mu_{0} \sigma_{c} \omega \varepsilon^{2} \ll 1$. The parameter $\omega$ is the frequency of the ablator and subindex $c$ denotes a characteristic value.

- Magnetic effects are not considered in Ohm's law, as $v B_{c} / E_{c} \ll 1$.

- Blood is considered as an incompressible and calorically perfect liquid.

- Blood perfusion is the dominant effect of external heat transfer [18], and it is modeled by means of Newton's law of cooling [19], while Fourier's law applies to the heat flux.

- All parameters are constant except from the electric conductivity of the cardiac tissue, $\sigma_{c t}=\sigma_{0}+$ $\sigma_{1}\left(T-T_{0}\right)$, which is a first order approximation of $\sigma_{c t}(t)=\sigma_{0} e^{\sigma_{1}\left(T-T_{0}\right) / \sigma_{0}}[20]$.

Taking into account the previous simplifications, the system of equations (1) - (6) reads:

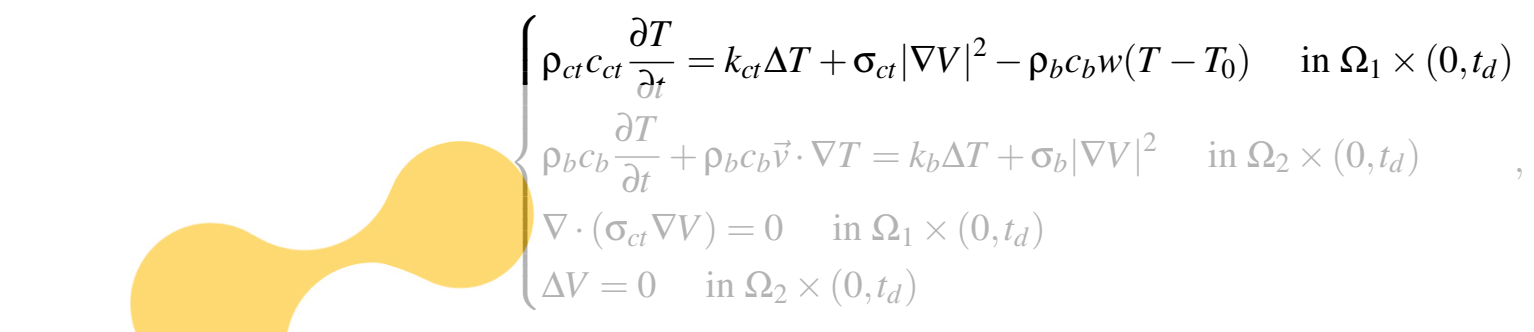

where $V$ is electric potential, $T$ temperature, $c$ specific heat capacity, $k$ thermal conductivity, $w$ perfusion frequency, $T_{0}$ human body temperature and $t_{d}$ discharge time. Subindex $b$ is referred to the blood.

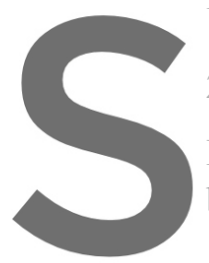

2.3 Initial and boundary conditions
Initial values for cardiac tissue and biood temperat
both values are the same and equal to human body
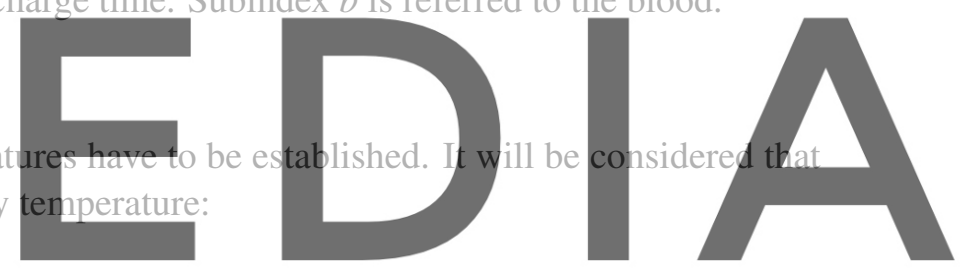

Register for free at https//www scipedia $T=T_{0} \quad$ in $\Omega \times\{t=0\}$.

(11)

Register for free at https//www.scipedia.com to download the version without the watermark

In regards to the boundary conditions, firstly, the symmetry condition at $\theta, \varphi=\pi / 2$ leads to:

$$
\frac{\partial T}{\partial \theta}=\frac{\partial V}{\partial \theta}=0 \quad \text { on } \partial \Omega_{1 s} \times\left(0, t_{d}\right) ; \quad \frac{\partial T}{\partial \varphi}=\frac{\partial V}{\partial \varphi}=0 \quad \text { on } \partial \Omega_{2 s} \times\left(0, t_{d}\right) .
$$

Considering that the catheter is refrigerated [21], its surface temperature is constant and equal to human body temperature:

$$
T=T_{0} \quad \text { on }\left(\partial \Omega_{11} \cup \partial \Omega_{22}\right) \times\left(0, t_{d}\right) .
$$

It will be assumed that when $r \rightarrow \infty$ the voltage vanishes, while the temperature on both boundaries is equal to human body temperature:

$$
T=T_{0} \text { and } V=0 \quad \text { on }\left(\partial \Omega_{1 \infty} \cup \partial \Omega_{2 \infty}\right) \times\left(0, t_{d}\right) .
$$


With respect to the voltage, defining $V_{0}$ as voltage amplitude and $\alpha=k /(\rho c)$ thermal diffusivity and assuming that $\alpha_{c t} \sim \alpha_{b}$ and $\alpha_{c} \omega / \varepsilon^{2} \gg 1$, root mean square value is imposed at $r=\varepsilon$ :

$$
V=\frac{V_{0}}{\sqrt{2}} \quad \text { on }\left(\partial \Omega_{11} \cup \partial \Omega_{22}\right) \times\left(0, t_{d}\right) .
$$

Finally, it is necessary to add four compatibility conditions on the cardiac tissue-blood interface: two for the thermal problem and two for the electrical problem. This will be done imposing temperature and voltage continuity:

$$
T \text { and } V \text { continuous } \quad \text { on }\left(\partial \Omega_{12} \cup \partial \Omega_{21}\right) \times\left(0, t_{d}\right),
$$

as well as equality of the heat and electric fluxes, where $\sigma_{c t} / \sigma_{b} \approx 1$ in order to avoid temperaturedependent boundary conditions:

$$
k_{c t} \frac{\partial T}{\partial \theta}=-k_{b} \frac{\partial T}{\partial \varphi} \text { and } \frac{\partial V}{\partial \theta}=-\frac{\partial V}{\partial \varphi} \quad \text { on }\left(\partial \Omega_{12} \cup \partial \Omega_{21}\right) \times\left(0, t_{d}\right)
$$

In view of the above, if $\beta=\theta$ or $\phi$ as appropriate, the system of equations for temperature reads:
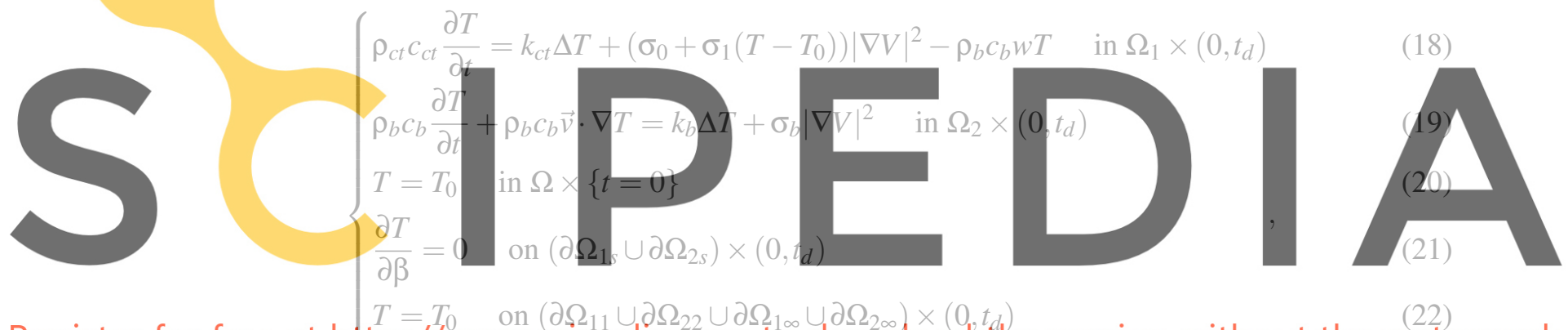

where $\beta$ is the polar coordinate $\theta$ or $\psi$, depending on wheter subdomain $\Omega_{1}$ or $\Omega_{2}$ is considered. Now, for the voltage the following model is considered:

$$
\left\{\begin{array}{l}
\nabla \cdot\left[\left(\sigma_{0}+\sigma_{1}\left(T-T_{0}\right)\right) \nabla V\right]=0 \quad \text { in } \Omega_{1} \times\left(0, t_{d}\right) \\
\Delta V=0 \quad \text { in } \Omega_{2} \times\left(0, t_{d}\right) \\
\frac{\partial V}{\partial \beta}=0 \quad \text { on }\left(\partial \Omega_{1 s} \cup \partial \Omega_{2 s}\right) \times\left(0, t_{d}\right) \\
V=\frac{V_{0}}{\sqrt{2}} \quad \text { on }\left(\partial \Omega_{11} \cup \partial \Omega_{22}\right) \times\left(0, t_{d}\right) \\
V=0 \quad \text { on }\left(\partial \Omega_{1 \infty} \cup \partial \Omega_{2 \infty}\right) \times\left(0, t_{d}\right) \\
V \text { continuous and } \frac{\partial V}{\partial \theta}=-\frac{\partial V}{\partial \varphi} \quad \text { on }\left(\partial \Omega_{12} \cup \partial \Omega_{21}\right) \times\left(0, t_{d}\right)
\end{array}\right.
$$




\subsection{Numerical implementation}

A Chebyshev spectral collocation method [22] is proposed for the spatial discretization of the system of equations, being Chebyshev-Gauss nodes the collocation points. Moreover, DGESV subroutine is used to solve the elliptic equations (24) - (29). It is part of the LAPACK library [23], and the numerical method is based on LU factorization. Finally, the temporal discretization of the system of equations (18) - (23) is done by means of a 4th order Runge-Kutta method [24].

\section{RESULTS}

In this section the results obtained by solving numerically the system of equations (18) - (29) are discussed. For that purpose, first of all, the results of a simulation of a case of practical application are shown in Subsection 3.1, including a discussion about the modeling of two parameters of the problem. Then, a parametric study is performed in Subsection 3.2.

\subsection{Case of practical application}

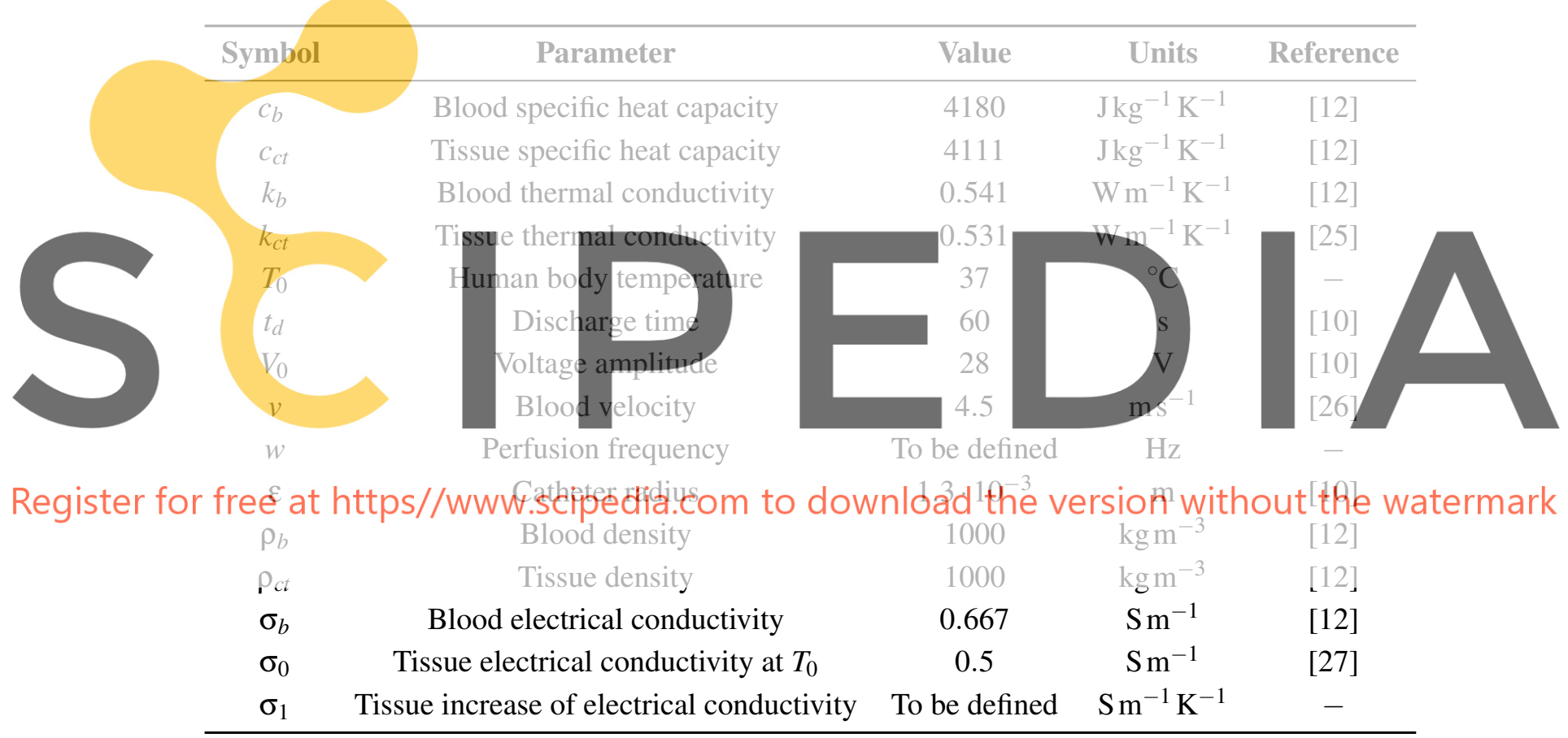

Table 1: Parameters of the model

The values of the parameters of the model, except from $w$ and $\sigma_{1}$, can be seen in Table 1 . There are multiple approaches available in the literature, so numerical simulations will be performed for different cases. The decision will be based on the values of three parameters:

1. $T_{\max }$ : Maximum temperature.

2. $r_{\max }$ : Location of maximum temperature relative to the surface of the catheter. 
3. $r_{\text {safe }}$ : Distance to $r=\varepsilon$ from which $T<40{ }^{\circ} \mathrm{C}$, due to the presence of the esophagus.

Furthermore, the numerical scheme has been validated, and a mesh independence study has been performed [28], leading to time step $\mathrm{d} t=2.5 \cdot 10^{-4} \mathrm{~s}$ and Chebyshev polynomial order $N=15$; that is, the number of elements of the mesh is $N^{2}=225$.

\subsubsection{Electrical conductivity}

Three different values of $\sigma_{1}$ are considered in the literature. Constant electrical conductivity for the cardiac tissue [14], $\sigma_{1}=0.015 \mathrm{~S} \mathrm{~m}^{-1} \mathrm{~K}^{-1}[11,12]$ and $\sigma_{1}=0.02 \mathrm{~S} \mathrm{~m}^{-1} \mathrm{~K}^{-1}[9,10]$. This is the reason why it is important to clarify the effect of $\sigma_{1}$ in the results of the numerical simulations. In addition, the case $\sigma_{1}=0$ is particularly interesting, as the elliptic equations can be solved analytically, leading to $V=V_{0} \varepsilon /(\sqrt{2} r)$. Perfusion is considered in these simulations with a value of $w=0.017 \mathrm{~Hz}$ [14].

\begin{tabular}{cccc}
\hline$\sigma_{1}\left(\mathrm{Sm}^{-\mathbf{1}} \mathbf{K}^{-\mathbf{1}}\right)$ & $\mathrm{T}_{\max }\left({ }^{\circ} \mathrm{C}\right)$ & $\mathrm{r}_{\max }(\mathrm{mm})$ & $\mathrm{r}_{\text {safe }}(\mathrm{mmm})$ \\
\hline 0 & 62.091 & 0.792 & 5.752 \\
0.015 & 67.051 & 0.791 & 6.502 \\
0.02 & 68.498 & 0.79 & 6.722 \\
\hline
\end{tabular}

Table 2: Influence of the model for electrical conductivity

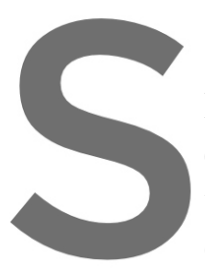

Based on the values presented in Table 2, it is necessary to consid
conductivity, as the maximum ternperature and the safe radius in the
wih respect to temperature-dependent models. Lower values of $T_{\mathrm{m}}$
of the equation of conservation of energy (18) is proportional to
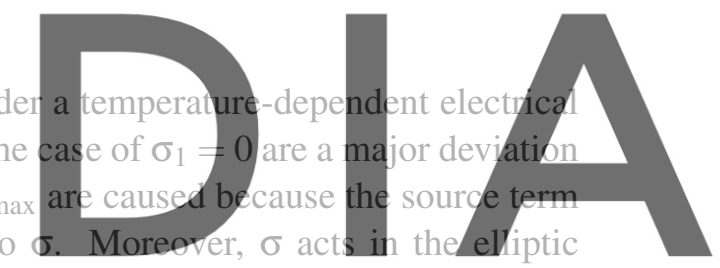

equation (23) as $k$ in the steady heat equation; as $\sigma$ increases, voltage distribution is smoother. The

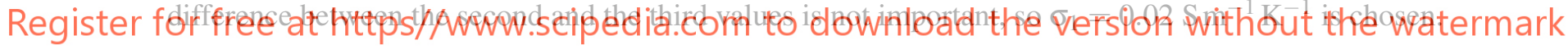

\subsubsection{Perfusion term}

Blood perfusion is the process where blood passes from the circulatory system to an organ or tissue through capillaries, in order to supply oxygen. In the case of catheter ablation it is a cooling process, so a term arises in the equation of conservation of energy, and, in principle, it is not clear if it is dominant or not. Most of publications neglect it as [10,12,13], while other authors consider it [14].

\begin{tabular}{cccc}
\hline $\mathbf{w}(\mathbf{H z})$ & $\mathbf{T}_{\max }\left({ }^{\circ} \mathbf{C}\right)$ & $\mathbf{r}_{\max }(\mathbf{m m})$ & $\mathbf{r}_{\text {safe }}(\mathbf{m m})$ \\
\hline 0.017 & 68.498 & 0.79 & 6.722 \\
0 & 78.696 & 0.896 & 8.506 \\
\hline
\end{tabular}

Table 3: Influence of perfusion term 
Table 3 suggests that blood perfusion is an effective method to prevent from important temperature variations in the cardiac tissue. This is because blood temperature is supposed to be equal to $37^{\circ} \mathrm{C}$ inside the capillaries, so the difference between tissue and blood temperatures is big, causing high rates of heat transfer. If the temperature of the cardiac tissue increases, the cooling process becomes more effective, and this is the reason why $r_{\text {safe }}$ is higher in the case of the absence of the perfusion term. Consequently, perfusion term is taken into account in the current model with $w=0.017 \mathrm{~Hz}$.

\subsubsection{Numerical results}

Once setting values of the parameters, first of all, Figures $2 \mathrm{a}-2 \mathrm{~d}$ illustrate that blood temperature increase is not important, while the maximum temperature is located near the surface of the catheter. At $t=60 \mathrm{~s}$, $T_{\max } \approx 68{ }^{\circ} \mathrm{C}$, which is far from the limit temperature, so there are no risks associated with the occurence of stream pops, while $r_{\max } \approx 0.8 \mathrm{~mm}$. Moreover, $r_{\text {safe }} \approx 6.7 \mathrm{~mm}$, which is higher than the approximate mean distance to the esophagus, $5 \mathrm{~mm}[4]$.

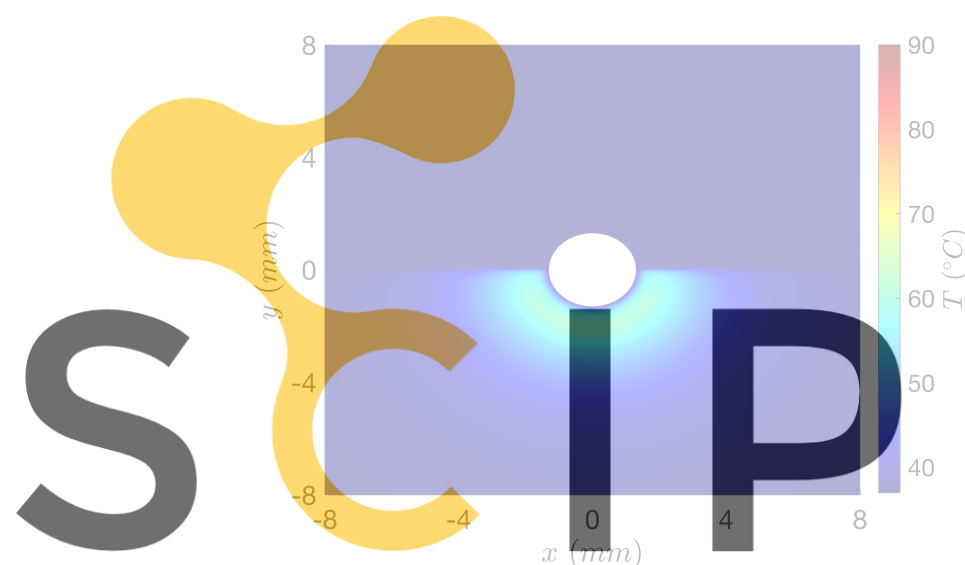

(a) $t=15 \mathrm{~s}$

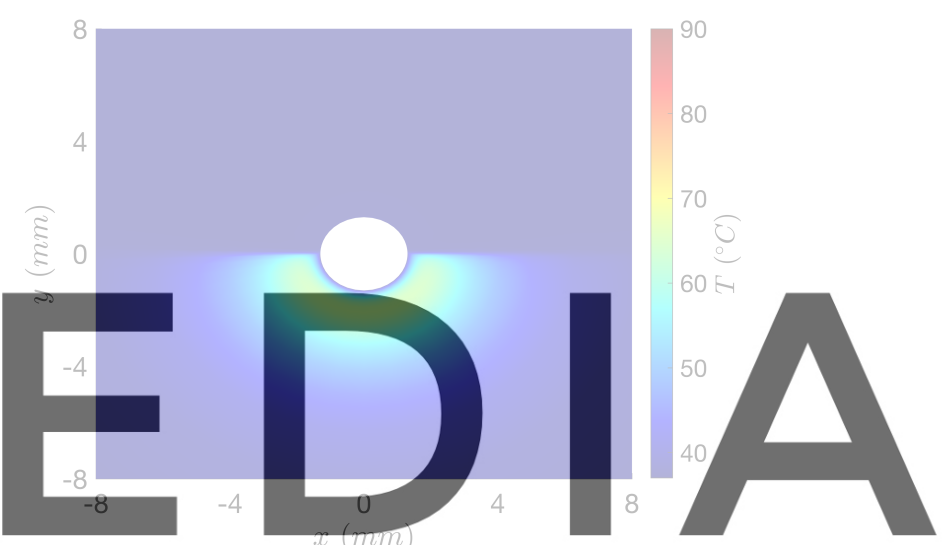

(b) $t=30 \mathrm{~s}$

Register for free at https//www.scipedia.com to download the version without the watermark

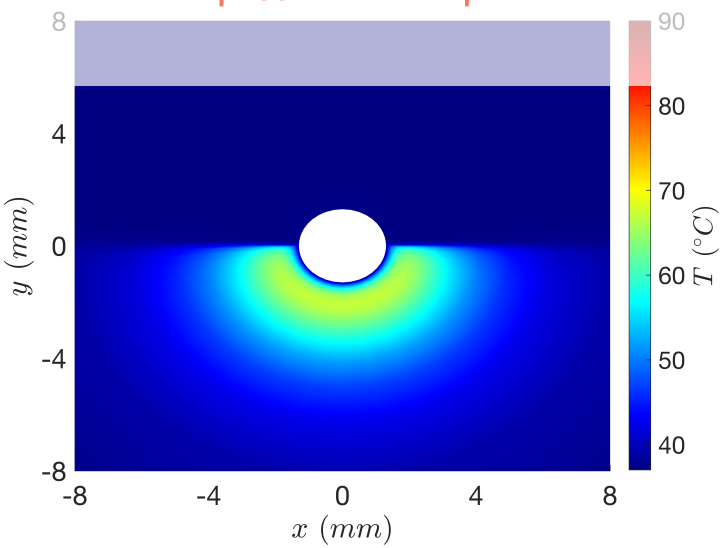

(c) $t=45 \mathrm{~s}$

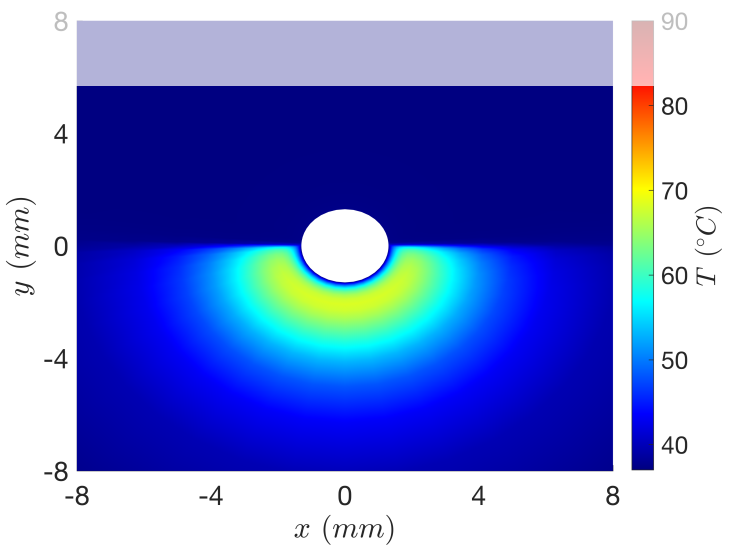

(d) $t=60 \mathrm{~s}$

Figure 2: Temperature distribution 


\subsection{Parametric study}

Focusing attention on the ablation procedure, three parameters involved in the model can be modified: discharge time $t_{d}$, voltage amplitude $V_{0}$ and catheter radius $\varepsilon$.

\subsubsection{Amplitude of the voltage and discharge time}

Firstly, the effect of the voltage and the discharge time will be analysed separately. On the one hand, it can be seen in Table 4 that the variations in maximum temperature and its location are negligible, while the safe radius increases significantly as time increases; that is, at large times the zones far from the catheter surface are the ones that suffer significant temperature changes.

\begin{tabular}{cccc}
\hline $\mathbf{t}(\mathbf{s})$ & $\mathbf{T}_{\max }\left({ }^{\circ} \mathbf{C}\right)$ & $\mathbf{r}_{\max }(\mathbf{m m})$ & $\mathbf{r}_{\text {safe }}(\mathbf{m m})$ \\
\hline 45 & 67.397 & 0.779 & 6.298 \\
60 & 68.498 & 0.79 & 6.722 \\
75 & 69.123 & 0.795 & 6.99 \\
\hline
\end{tabular}

Table 4: Influence of discharge time $\left(V_{0}=28 \mathrm{~V}\right)$

On the other hand, another variable parameter is voltage amplitude $V_{0}$. Considering three different values, Figure 3 shows that the curves of maxinum te
to mention that small differences in $V_{0}$ give rise to im
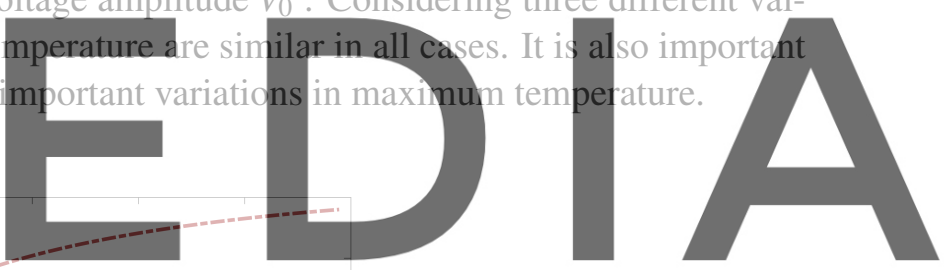

Register for free at https//www.scipedix.com to downłoad the- version without the watermark

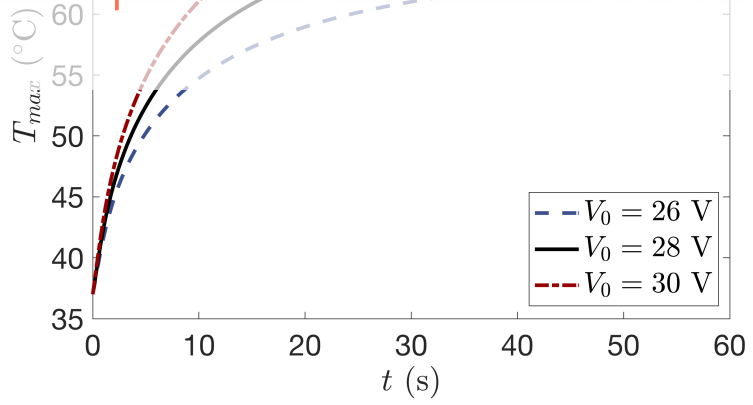

Figure 3: Maximum tissue temperature for different values of voltage amplitude

Finally, a combination of both variations can also be done. If $T_{\max }$ is set to be approximately constant, then it can be verified in Table 5 that higher values of $V_{0}$ produce temperature distributions that are more concentrated near the catheter. Consequently, if the esophagus is near the endocardium, the best choice is to increase the voltage amplitude and reduce the discharge time as required. 


\begin{tabular}{ccccc}
\hline $\mathbf{t}(\mathbf{s})$ & $\mathbf{V}_{\mathbf{0}}(\mathbf{V})$ & $\mathbf{T}_{\max }\left({ }^{\circ} \mathbf{C}\right)$ & $\mathbf{r}_{\mathbf{m a x}}(\mathbf{m m})$ & $\mathbf{r}_{\text {safe }}(\mathbf{m m})$ \\
\hline 30 & 29 & 67.797 & 0.754 & 5.817 \\
60 & 28 & 68.498 & 0.79 & 6.722 \\
90 & 27 & 66.789 & 0.801 & 6.917 \\
\hline
\end{tabular}

Table 5: Influence of discharge time and voltage amplitude

\subsubsection{Catheter radius}

The last parameter of the procedure that can be modified is catheter radius. As the catheter has to be inserted through a vein, there will be a maximum permissible value, while the catheter is not supposed to be too small because of design limitations, so minor modifications will be considered.

\begin{tabular}{ccccc}
\hline$\varepsilon(\mathrm{mm})$ & $\mathrm{T}_{\max }\left({ }^{\circ} \mathrm{C}\right)$ & $\mathrm{r}_{\max }(\mathrm{mmm})$ & $\mathrm{r}_{\text {safe }}(\mathrm{mmm})$ \\
\hline 1.1 & 72.137 & 0.703 & 6.548 \\
1.3 & 68.498 & 0.79 & 6.722 \\
1.5 & 65.575 & 0.872 & 6.875 \\
\hline
\end{tabular}
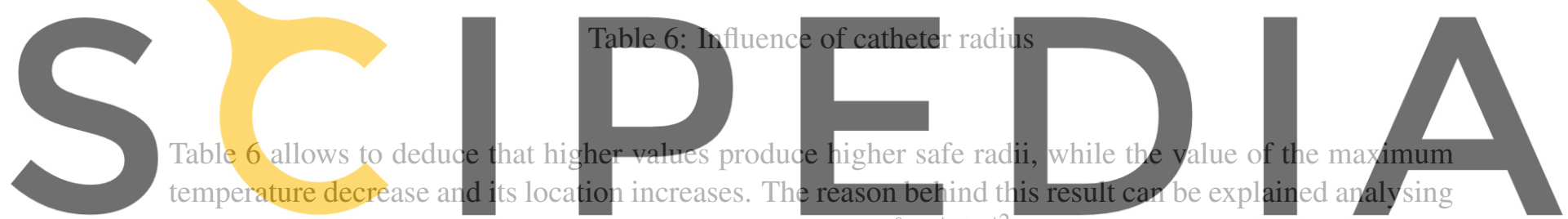

the right hand side equation (18). The applied power $P=\int_{\Omega} \sigma|\nabla V|^{2}$, and making use of Stokes theorem

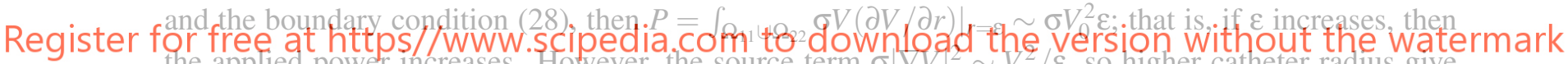
the applied power increases. However, the source term $\sigma|V| \sim V_{0}^{2} / \varepsilon$, so higher catheter radius give rise to a smoother voltage distribution. This variation leads to lower values of the source terms near the surface of the catheter, as the heat flux due to perfusion $q_{p} \sim \rho c w T$ does not depend on $\varepsilon$.

\section{CONCLUSIONS}

In this work a mathematical model has been developed to study catheter ablation procedure. The obtained results have shown that a temperature-dependent electrical conductivity of the cardiac tissue is necessary, and it has been deduced that blood perfusion is an effective cooling process, even though further research is needed concerning the modeling of this term. A case of practical application has been simulated, giving rise to maximum temperatures that are far from dangerous values, even though temperature values should be reduced near the esophagus. A parametric study has been performed, showing that high values of the voltage and small values of catheter radius and discharge time are the best choice for safe ablations.

The work presented here provides a basis for the modeling of catheter ablation. As a first step towards a more accurate model, the axisymmetry assumption can be relaxed, allowing to better understand the blood flow, as well as to study the influence of the contact force between the catheter and the tissue. 


\section{REFERENCES}

[1] Benjamin, E.J., Muntner, P., Alonso, A., Bittencourt, M.S. et al. Heart Disease and Stroke Statistics - 2019 Update: A Report From the American Heart Association. AHA Journal of Circulation (2019) 139(10).

[2] Calkins, H., Hindricks, G., Cappato, R., Kim, Y-H. et al. RS/EHRA/ECAS Expert Consensus Statement on Catheter and Surgical Ablation of Atrial Fibrillation. Europace (2012) 14:528-606.

[3] Cochet, H., Sacher, F., Chaumeil, A. and Jais, P. Steam Pop During Radiofrequency Ablation. Imaging Features on Magnetic Resonance Imaging and Multidetector Computed Tomography. Circulation: Arrhythmia and Electrophysiology (2014) 7(3):559-560.

[4] Sánchez-Quintana, D., Cabrera, J.A., Climent, V, Farré, J. et al. Anatomic Relations Between the Esophagus and Left Atrium and Relevance for Ablation of Atrial Fibrillation. AHA Journal of Circulation (2005) 112(10):1400-1405.

[5] Halm, U., Gaspar, T., Zachäus, M., Sack, S. et al. Thermal Esophageal Lesions After Radiofrequency Catheter Ablation of Left Atrial Arrhythmias. American Journal of Gastroenterology (2010) 105(3):551-556.

[6] Anfuso, L., Corsi, M and Fasano, A. Esophageal Thermal Probes: How Fast Should They Be? Matthews Journal of Cardiology (2018) 3(1):1-5.

[7] Gianni, C., Atoui, M., Mohanty, S., Trivedi, C. et al. Difference in Thermodynamics Between Two Types of Esophageal Temperature Probes: Insights from an Experimental Study. Heart Rhythm (2016) 13(11):2195-2200.

[8] Knecht, S., Sticherling, C., Reichlin, T., Mühl, A. et al. Reliability of Luminal Oesophageal Temperature Monitoring During Radiofrequency Ablation of Atrial Fibrillation: Insights from Probe Visualization and Oesophageal Reconstruction Using Magnetic Resonance Imaging. Europace (2017) 19(7):123-1131.

[9] Kaouk, Z., Vahid Shahidi, A., Savard, P. and Molin, F. Modelling of Myocardial Temperature Distribution During Radio-Frequency Ablation. Medical \& Biological Engineering \& Computing (1996) 34(2):165-170.

[10] Tungjitkusolmun, S., Woo, E.J., Cao, H., Tsai, J.Z. et al. Thermal - Electrical Finite Element Modelling for Radio Frequency Cardiac Ablation: Effects of Changes in Myocardial Properties. Medical \& Biological Engineering \& Computing (2000) 38(2):562-568.

[11] Schutt, D., Berjano, E. and Haemmerich, D. Effect of Electrode Thermal Conductivity in Cardiac Radiofrequency Catheter Ablation: A Computational Modeling Study. International Journal of Hyperthermia (2009) 25(2):99-107.

[12] González-Suárez, A., Pérez, J.J. and Berjano, E. Should Fluid Dynamics be Included in Computer Models of RF Cardiac Ablation by Irrigated-Tip Electrodes? Biomedical Engineering Online (2018) 17(1).

[13] Petras, A., Leoni, M., Guerra, J.M., Jansson, J. et al. A Computational Model of Open-Irrigated Radiofrequency Catheter Ablation Accounting for Mechanical Properties of the Cardiac Tissue. International Journal of Numerical Methods in Biomedical Engineering (2019) 35(11). 
[14] Fasano, A., Anfuso, L., Bozzi, S. and Pandozi, C. Safety And Necessity Of Thermal Esophageal Probes During Radiofrequency Ablation For The Treatment Of Atrial Fibrillation. Journal of Atrial Fibrillation (2016) 9(1):11-18.

[15] Haïssaguerre, M., Jaïs, P., Shah, D.C., Takahashi, A. et al. Spontaneous Initiation of Atrial Fibrillation by Ectopic Beats Originating in the Pulmonary Veins. The New England Journal of Medicine (1998) 339(10):659-666.

[16] Landau, L.D. and Lifshitz, E.M. The Classical Theory of Fields. Butterworth-Heineman, Vol. I. (1980).

[17] Landau, L.D. and Lifshitz, E.M. Fluid Mechanics. Butterworth-Heineman, Vol. VI. (1987).

[18] Berjano, E.J. Theoretical Modeling for Radiofrequency Ablation: State of the Art and Challenges for the Future. Biomedical Engineering Online (2006) 5(24).

[19] Charny, C.K. Mathematical Models of Bioheat Transfer. Advances in Heat Transfer (1992) 22:19155.

[20] Duck, F.A. Physical Properties of Tissues. A Comprehensive Reference Book. Academic Press (1990).

[21] Reiter, T., Gensler, D., Ritter, O., Weiss, I. et al. Direct Cooling of the Catheter Tip Increases Safety for CMR-Guided Electrophysiological Procedures. Journal of Cardiovascular Magnetic Resonance (2012) 14(12).

[22] Kopriva, D.A. Implementing Spectral Methods for Partial Differential Equations. Springer (2009).

[23] The Netlib. LAPACK Documentation.

[24] Butcher, J.C. Numerical Methods for Ordinary Differential Equations. Wiley (2016).

[25] Valvano, J.W. and Bhabaraju, J.C. Thermophysical Properties of Swine Myocardium. International Journal of Thermophysics (1999) 20(2):665-676.

[26] Benchimol, A., Desser, K.B. and Gartlan, J.C. Bidirectional Blood Flow Velocity in the Cardiac Chambers and Great Vessels Studied with the Doppler Ultrasonic Flowmeter. The American Journal of Medicine (1972) 54(4):467-473.

[27] Forster, K.R. and Schwan, H.P. Dielectric Properties of Tissues and Biological Materials: A Critical Review. Critical Reviews in Biomedical Engineering (1989) 17(1):25-104.

[28] Amatriain, A. Mathematical Modeling and Numerical Simulation of Catheter Ablation Procedure. Master's Thesis (2019). https://eprints.ucm.es/id/eprint/63176/ 